\title{
Classic Linear Methods Provisos Verification for Oscillator Design Using NDF and Its Use as Oscillators Design Tool
}

\author{
Angel Parra-Cerrada, Vicente González-Posadas, José Luis Jiménez-Martín, Alvaro Blanco \\ Department Ingeniería Audiovisual y Comunicaciones, Universidad Politécnica de Madrid, Madrid, Spain \\ Email: angelpa@diac.upm.es
}

Received September 22, 2012; revised December 22, 2012; accepted December 30, 2012

\begin{abstract}
The purpose of this paper is to show the conditions that must be verified before use any of the classic linear analysis methods for oscillator design. If the required conditions are not verified, the classic methods can provide wrong solutions, and even when the conditions are verified each classic method can provide a different solution. It is necessary to use the Normalized Determinant Function (NDF) in order to perform the verification of the required conditions of the classic methods. The direct use of the NDF as a direct and stand-alone tool for linear oscillator design is proposed. The NDF method has the main advantages of not require any additional condition, be suitable for any topology and provide a unique solution for a circuit with independence of the representation and virtual ground position. The Transpose Return Relations $\left(R R_{T}\right)$ can be used to calculate the NDF of any circuit and this is the approach used to calculate the NDF on this paper. Several classic topologies of microwave oscillators are used to illustrate the problems that the classic methods present when their required conditions are not verified. Finally, these oscillators are used to illustrate the use and advantages of the NDF method.
\end{abstract}

Keywords: Oscillator; Provisos; NDF; RR; $R R_{T}$

\section{Introduction}

The oscillators are one of the most important circuit types on nowadays for communication systems and, due to its non-linearity, they are one of the circuits that have more problems on their design and optimization process. The linear simulation of these circuits is really important due to it is suitable for circuit optimization [1-3] and it needs less computational resources than the non-linear simulation. In any case, even if the designer is going to use a non-linear simulation, it is necessary to perform a linear simulation to have a good approximation of oscillation frequency before starting the non-linear simulation. But to be sure that the linear methods provide good solutions, there are some provisos that must be verified.

\section{Classic Methods Provisos}

The classic methods for linear analysis of oscillators can be classified into two groups: reference plane methods $[1,4]$ and the gain-loop method [5]. The provisos for each group of methods are described in the following sections.

This paper describes the main conclusions and the necessary provisos for the proper use of the classic methods; they are defined and justified in detail by the authors [6-8].

\subsection{Reference Plane Methods}

Any oscillator may be analyzed using $Z, Y$ or $\Gamma$ network functions (Figure 1), in some cases it is more convenient to use a specific method, but any of the reference plane methods can be used. It is important to remember that all the system poles are included on any network function; however all the poles are not always included on general transfer functions. The necessary condition for a circuit to be a proper oscillator is that it must only have a pair of complex conjugated poles in the Right Half Plane (RHP).

The traditional drawing way is conditioned in order to

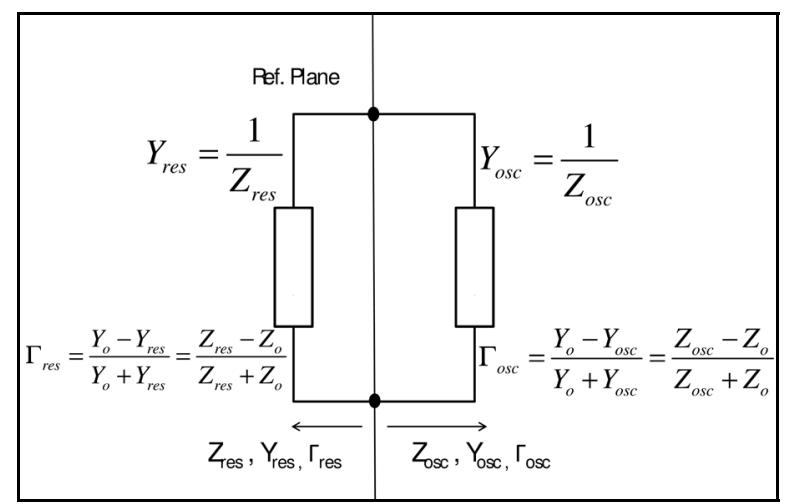

Figure 1. Oscillator as two subsystems. 
find the resonant structure as a dipole isolated from the negative $Z / Y / \Gamma$ generator. The reference plane can be any (Figure 1), without being a real division between resonator and generator; it is possible thanks to the denominator of any network function has all the information about the system poles. But using one of the traditional divisions simplifies the necessary conditions to assure a correct linear analysis. These classic methods are really the application of the Nyquist's criteria for resolving the location of poles in the RHP of the network functions.

\subsection{Negative Conductance Analysis (Impedance Network Function )}

Figure 2 presents the conceptual diagram for a negative conductance analysis. The impedance network function is defined by Equation (1), where $I_{g}$ is the external current; $V$ is the circuit response; and $Z$ is the inverse of the sum of the admittances of Figure 2. The circuit is a proper oscillator if the network function has only a pair of conjugated complex poles in the RHP. The poles of the network function are defined by the zeros of Equation (2), which is the characteristic function of the circuit. Then, Equation (2) is analysed with the Nyquist criteria.

$$
V=Z \cdot I_{g}
$$

where:

$$
\begin{aligned}
& Z=1 /\left(Y_{\text {res }}+Y_{\text {osc }}\right) \\
& Y_{T}=Y_{\text {res }}+Y_{\text {osc }}=0
\end{aligned}
$$

The classic oscillator start-up condition, $\mathfrak{I}\left(Y_{T}\right)=\mathfrak{I}\left(Y_{\text {res }}+Y_{\text {osc }}\right)=0$ and $\mathfrak{R}\left(Y_{T}\right)=\mathfrak{R}\left(Y_{\text {res }}+Y_{\text {osc }}\right)<0$ is a simplification of the Nyquist analysis to a condition for a single frequency, but it is not sufficient condition to guarantee the start-up of the oscillator [6]. The additional condition before analyzing an oscillator with the negative conductance method (Impedance Network Function) is to assure that $Y_{\text {osc }}$ does not have any poles (visible or hidden) in the RHP. This verification uses the Normalized Determinant Function (NDF) [9] of a network built with the active sub-circuit terminated with a short-circuit, as it is de-

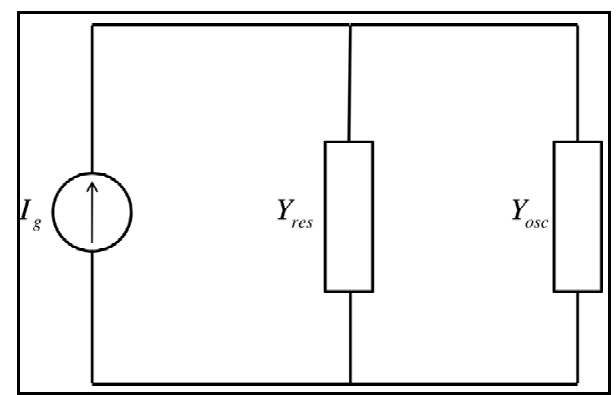

Figure 2. Negative conductance method conceptual diagram. scribed by Jackson based on Platzker and Ohtomo papers [9-11]. Each clockwise turning circle around the origin of the NDF analysis, for positive frequencies, confirms the existence of a pair of conjugated poles. As NDF has an asymptotic response with frequency to +1 , the upper analysis frequency is easily determined. So, the Nyquist analysis of the NDF of the active subnetwork loaded with a short-circuit must not encircle the origin, then the negative conductance analysis of the oscillator can be performed with guarantee. With this condition the Nyquist analysis of the Equation (2) will predict the oscillation if it has a unique encircle of the origin.

The NDF can be calculated by means of the Return Relations (RR) as it was described by Platzker and in a most suitable way using the Transpose Return Relations $\left(R R_{T}\right)$ [7], Equation (3). The formulation of the NDF which uses the $R R_{T}$ is the one used by the authors to analyze the examples. To use the $R R_{T}$, it is necessary to replace the active devices with their linear models, then the $R R_{T i}$ term is the Transpose Return Relation for the $i$-depending generator while previous $i-1$ ones have been disabled.

$$
N D F=\frac{\Delta(s)}{\Delta_{0}}=\prod_{i=0}^{n}\left(R R_{i}+1\right)=\prod_{i=0}^{n}\left(1-R R_{T_{i}}\right)
$$

Negative Impedance Analysis (Admittance Network Function).

The oscillators to be analyzed with the $Y$ network function must also guarantee an additional proviso in the same way as the ones to be analyzed with the $Z$ network function. This proviso also makes use of the NDF analysis. The $Y$ network function is analyzed by means of the $Z$ characteristic function, then the Nyquist analysis of the NDF of the active sub-circuit loaded with an open-circuit must not encircle the origin. With this condition the Nyquist analysis of the Equation (4) will predict the oscillation if it has a unique encircle of the origin [6].

$$
Z_{T}=Z_{\text {res }}+Z_{\text {osc }}=0
$$

Negative Conductance Analysis (Impedance Network Function).

The $\Gamma$ network function must satisfy that the Nyquist analysis of the NDF of the $Z_{o}$ loaded active sub-circuit must not encircle the origin.

Then, the oscillation condition can be determined by the analysis of the Equation (5), so the oscillation is satisfied if the Nyquist analysis of $1-\Gamma_{\text {res }} \cdot \Gamma_{\text {osc }}$ encircles clockwise the 0 or if the Nyquist analysis of $\Gamma_{\text {res }} \cdot \Gamma_{\text {osc }}$ encircles clockwise the $+1[6]$.

$$
\Gamma_{T}=1-\Gamma_{\text {res }} \cdot \Gamma_{\text {osc }}=0
$$

\subsection{Loop-Gain Method}

When the feedback path of the circuit can be identified, the Loop-Gain is commonly used [3]. When it is possible 
to define the feedback path, this method is preferred by the designers because it is more intuitive and it can provide more useful information about the circuit. The start point for the loop-gain analysis is the general function of a loopback system (Figure 3), and the most important equation used nowadays is the one defined by Randall and Hock (Equation (6)) [5].

$$
G_{L}=\frac{Z_{21}-Z_{12}}{Z_{11}+Z_{22}-2 Z_{12}}=\frac{S_{21}-S_{12}}{1-S_{11} S_{22}+S_{12} S_{21}-2 S_{12}}
$$

The Nyquist analysis of the loop-gain $\left(G_{L}\right)$ must search for +1 clockwise encirclement to assure a proper oscillation condition, but the poles of the system must be only from the zeros of $1-G_{L}$. To guarantee that the poles of the system only come from the zeros of $1-G_{L}$ it is necessary that [7]:

None of the S parameters can have any poles in the RHP. This condition can be verified with the Nyquist analysis of the NDF of the open-loop circuit loaded with $Z_{o}$ in both ports.

"Test function" $T F=1-S_{11} S_{22}+S_{12} S_{21}-2 S_{12}$ must not have any zeros in the RHP. This condition is verified with the Nyquist analysis of TF when the condition of the previous point has been satisfied.

\section{Calculus Using the $\boldsymbol{R R}_{T}$}

The NDF can be easily calculated with the $R R_{T}$ of each active device using the Equation 3 as it was described by Platzker [9]. The first step for the $R R_{T}$ simulation of a transistor is to have a linear model of the device. If this linear model is not available for the transistor the parameters can be extracted with an "annotate" from the spice model with the AWR simulation software.

Once the linear model of the transistor is available, the next step is to calculate its $R R_{T}$. It can be calculated by making the transistor work as an independent AC Cur- rent Source (ACCS) with a constant value for all frequencies and with a voltage meter that measures the voltage at the control point of the real linear transistor model, Figure 4.

The same process must be performed for each transistor, but the ACCS of the already analyzed ones must be disabled before starting the simulation of the $R R_{T}$ of the next one.

Some commercial simulation software, as for example AWR, provides NDF function, but it is also possible to calculate $R R_{T}$ with any simulator. $R R_{T}$ is $-R R$ and it is "the true loop gain". The authors did not use the AWR NDF function for the simulations presented in this paper, but they used a general function based on the simulation of the $R R_{T}$ of each transistor.

\section{NDF as Oscillators Design Tool}

The main conclusion that can be obtained from the study of provisos for the classic linear oscillator design methods is that it is necessary to use the NDF to guarantee the applicability of any method. So, as it is described by the authors [8] the NDF is itself an interesting linear design method for oscillators design.

The NDF is the quotient of the network determinant and the normalized network determinant, Equation (3). The normalized network determinant is obtained by disabling all the active devices of the network, but it is easily solved by using the $R R_{T}$, as it has been described in the previous section.

The Nyquist plot analysis of the NDF determines in a single step the number of poles in the RHP of a network. Each clockwise encirclement of the origin for positive frequencies indicates the existence of a pair of conjugated complex poles in the RHP. So, the total phase evolution for a proper oscillator of the NDF for positive frequencies from $0 \mathrm{~Hz}$ up to $\infty \mathrm{Hz}$ must be $-360 \mathrm{deg}$. The

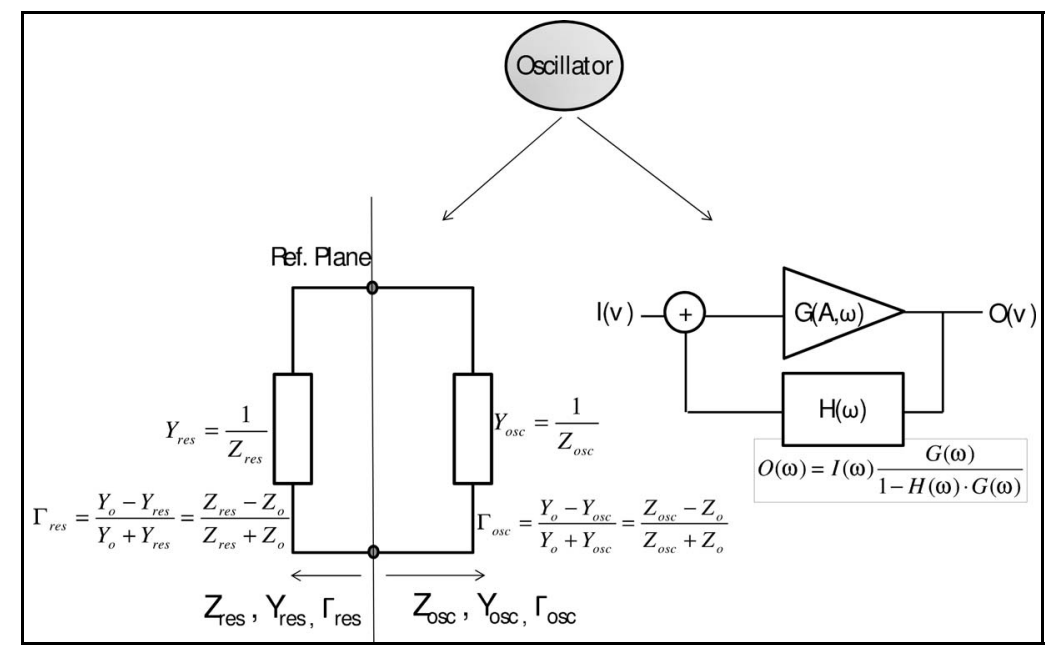

Figure 3. Reference plane method (left) and Feedback scheme (right). 


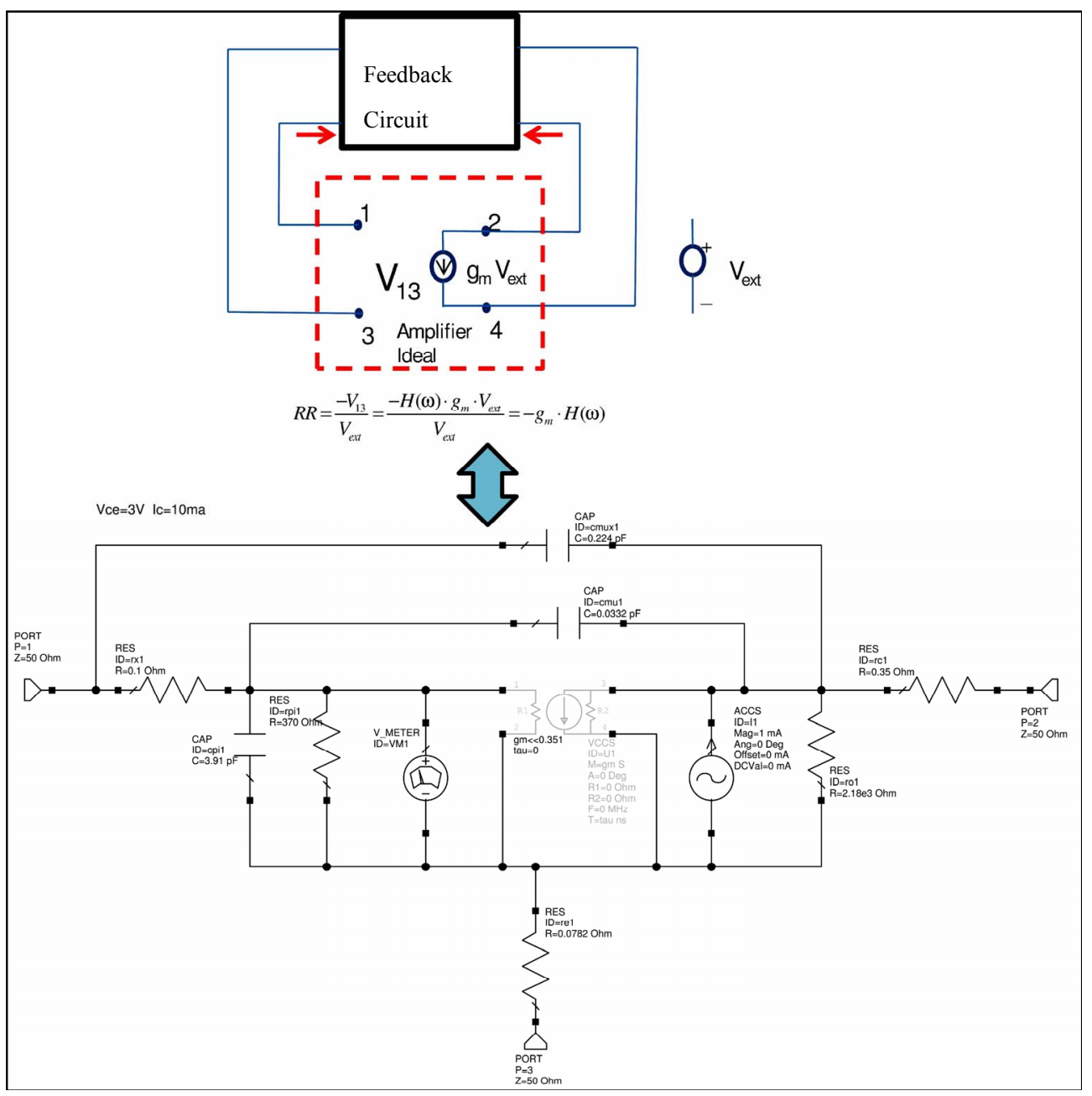

Figure 4. Simulation of the $R R_{T}$ of a transistor.

NDF has an asymptotic behavior towards +1 which is useful for determining the analysis upper frequency limit without ambiguity. As the NDF can be applied to any oscillator topology, it is a universal tool for analyzing oscillators on a single step.

Other useful characteristics of the NDF are that it can predict the oscillation frequency without transistor compression $\left(g_{m}\right)$ for Kurokawa's first harmonic approximation; and that it is suitable for the calculus of the $Q_{L}$ of the circuit because it is directly related with the $R R_{T}$ [8]. These characteristics make it suitable to use it as an optimization tool for low noise oscillators [12]. In the same way it is also suitable for estimating the start-up time. These two parameters, phase-noise and start-up time, are proper to the loop-gain method, but also to the NDF method. This way the NDF method makes available for any oscillator topology the parameters that until now were only available for the topologies that can be analysed with the loop-gain method, and on a single step and without ambiguity. This method is suitable for calculateing the real $Q_{L}$ factor of the circuit and the gain margin, so it is possible to estimate the start-up time and the phase noise of any oscillator without dependence with its topology.

\section{Examples}

Two examples are presented on this paper, one oscillator circuit which is usually analyzed with a reference plane method and another that is usually analyzed with the loop-gain method. These two examples have been chosen to illustrate the importance of the verification of the provisos and the advantage of using the NDF as an oscillator linear design tool. The circuits use as active device a BFR360F transistor biased with $I_{C}=10 \mathrm{~mA}$ and $V_{C E}=3 \mathrm{~V}$. The AWR software has been used for all the simulations shown in this paper.

\subsection{Example A}

The common collector oscillators, Figure 5, are usually analyzed by negative resistance, due it has the behaviour of a negative resistance generator for its first harmonic 
approximation response. The Nyquist representations of the total impedance and admittance of the example circuit are shown in Figures 6(a) and (b).

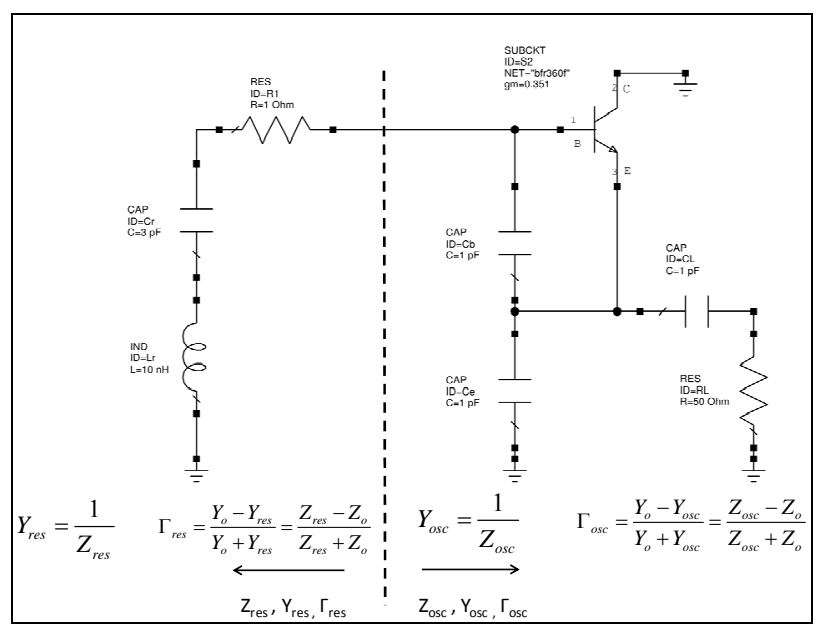

Figure 5. Common collector oscillator.

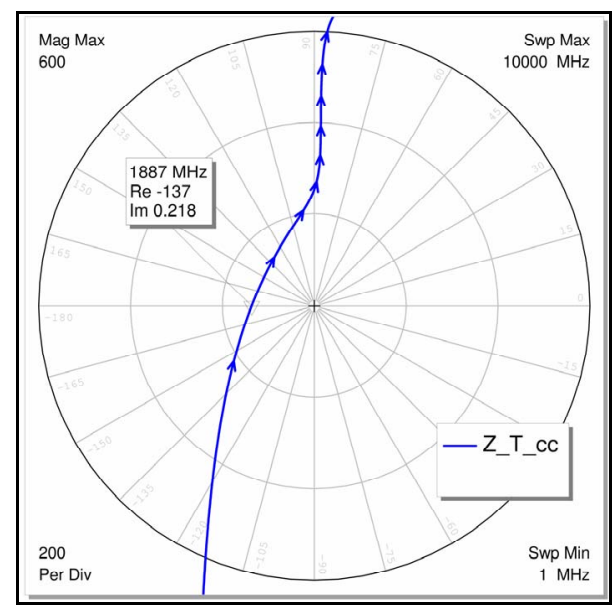

(a)

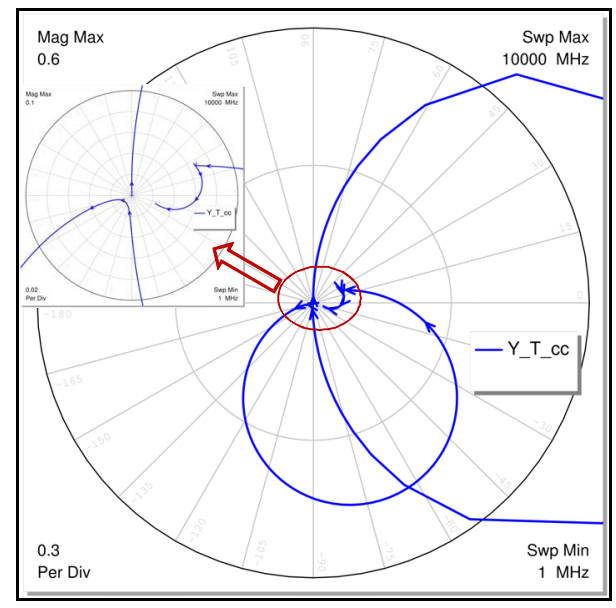

(b)

Figure 6. (a) Common collector oscillator total impedance and (b) total admittance.
The Nyquist analysis of $Z_{T}$, Figure 6(a), predicts an oscillation frequency of $1887 \mathrm{MHz}$, but the Nyquist analysis of $Y_{T}$, Figure 6(b), does not predict any oscillation condition. It can be explained because $Y_{\text {osc }}$ has a conjugated pair of poles in the RHP that hide the zeros on the Nyquist analysis of $Y_{T}$. This discrepancy points out the importance of the verification of the provisos before performing any classic analysis to an oscillator.

The Nyquist plot of the NDF of the active sub-circuit loaded with an open-circuit, Figure 7(a), does not encircle the origin, so it is possible to analyze this oscillator using the $Y$ network function (Nyquist analysis of the $Z_{T}$ ). But the NDF of the short-circuit loaded active sub-circuit, Figure 7(b), encircles the origin, $Y_{\text {osc }}$ has a pair of poles that will hide the zeros when the Nyquist criteria is used with the $Y_{T}$. Then the oscillator cannot be analyzed by the $Z$ network function (admittance analysis).

As an example of the use of the NDF, the NDF analysis of this circuit, Figure 8 encircles the origin and predicts an oscillation at $1474 \mathrm{MHz}$. The difference of os-

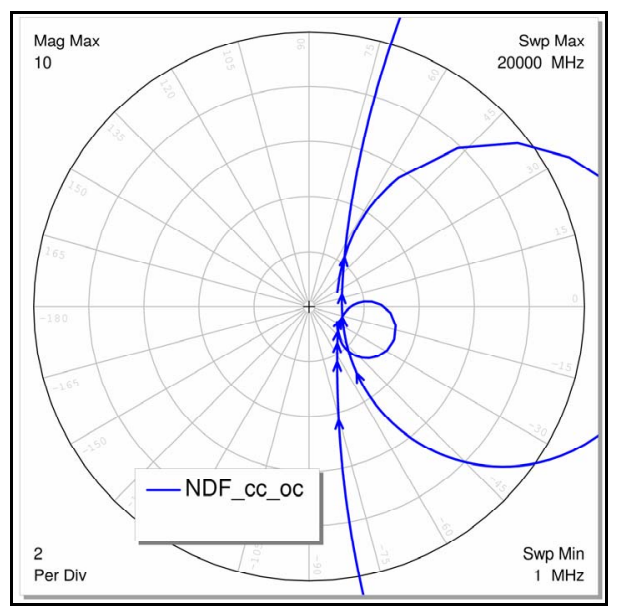

(a)

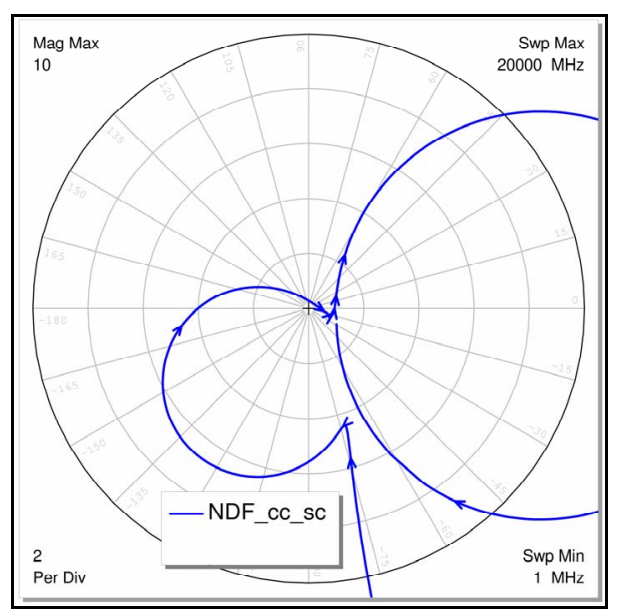

(b)

Figure 7. NDF of the (a) open-circuit and (b) short-circuit of 
the active sub-circuit.

cillation frequency is caused because the $Z_{T}$ method does not provided the first harmonic approximation but the NDF solves the first harmonic approximation without transistor compression. If the transistor is compressed to $g_{m}=0.0125$ then the $Z_{T}$ solution is the same that the NDF one, Figure 9.

As it has been shown with this example, an important advantage of using the NDF method is that it can be used with all oscillator topologies. In this example an oscillator that is usually analyzed with a reference plane method has been presented, so the main parameters that the reference plane methods cannot provide are now. The NDF is a "loop-gain concept" and it provides the first harmonic approximation without transistor compression. The $Q_{L}$ of the oscillator obtained with Equation (7) [12] is 9.3, Figure 10, and the gain margin is defined by the real part of NDF at the crossing point of the encircle of the origin. The big difference between the frequency ob-

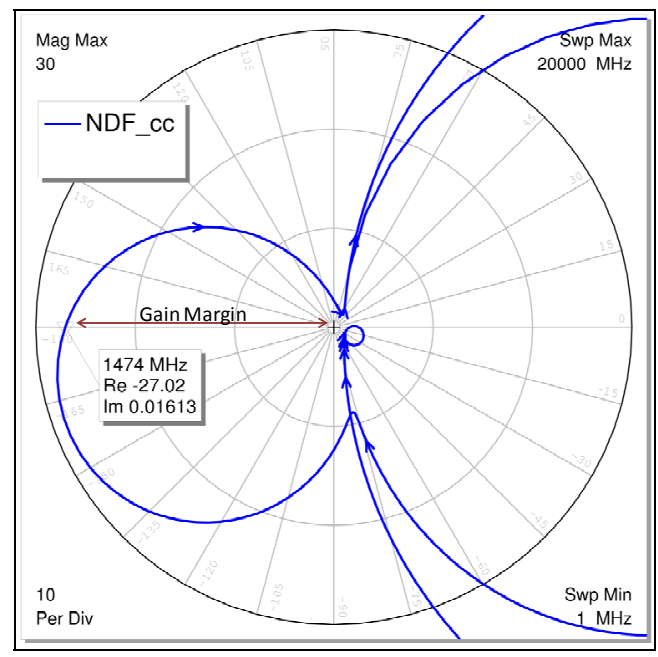

Figure 8. NDF of the common collector oscillator.

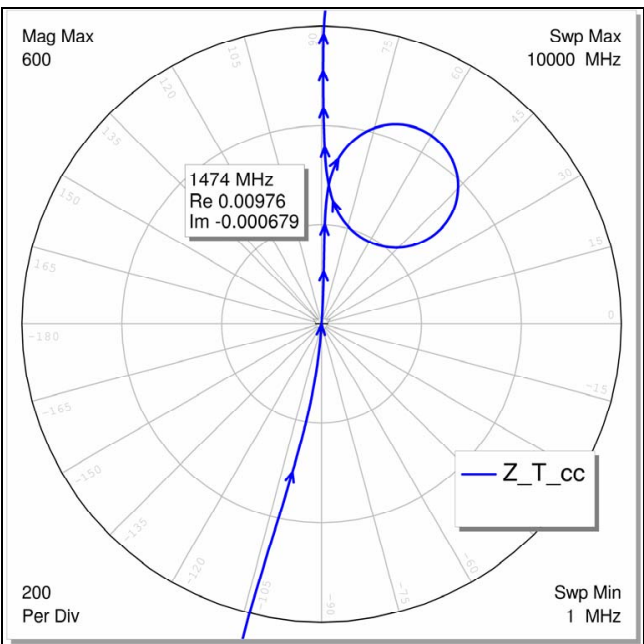

Figure 9. Common collector oscillator total impedance with $\boldsymbol{g}_{m}=\mathbf{0 . 0 1 2 5}$.

tained by the $Z_{T}$ without compression and the one obtained by the NDF is due to the low $Q_{L}$ of the oscillator.

$$
Q_{L}=-\frac{\omega}{2} \cdot \frac{\partial}{\partial \omega} \operatorname{Arg}\left(R R_{T}(\omega)\right)
$$

\subsection{Example B}

An oscillator, Figure 11(a), to which different virtual ground points [13] are applied, is used for this example. Some resulting possibilities of this example are the wellknown classic topologies: common collector (also named Colpitts), common emitter (also named Pierce) and common base. Using virtual ground concept, it is demonstrated that there is a unique oscillator topology and, as it will be explained throughout this example that the $N D F / R R_{T}$ is the best tool to analyze it. The circuits in Figure 11 include the parasites of the package of all devices, but these parasites have not been represented for readability.

The open-loop analysis, Figure 12, predicts that only the common emitter topology will oscillate, but "How can it be possible if the three schematics represent the same circuit?". The problem appears due to the Nyquist analysis of $G_{L}$ expression is not valid for common collector and common base topologies. In these two cases the denominators of $G_{L}$ have two hidden zeros which make Nyquist analysis not encircle +1 . It is interesting to point out that the $G_{L}$ analysis of the common base circuit complies the Barkhausen criteria at two different frequencies, $1131 \mathrm{MHz}$ and $3825 \mathrm{MHz}$. The first one crosses from a positive to a negative phase, but the second one crosses from a negative to a positive phase. The +1 is not encircled on the common collector example, neither on the common base, so they can be considered as complementary to Nguyen examples [14].

On the other hand, the NDF (or the $R R_{T}$ ), analyses have a unique solution for the three schematics, Figure 13. As all the NDF analyses are identical and they predict a unique complex pair of poles in the RHP, so the required condition for proper oscillation is satisfied for the

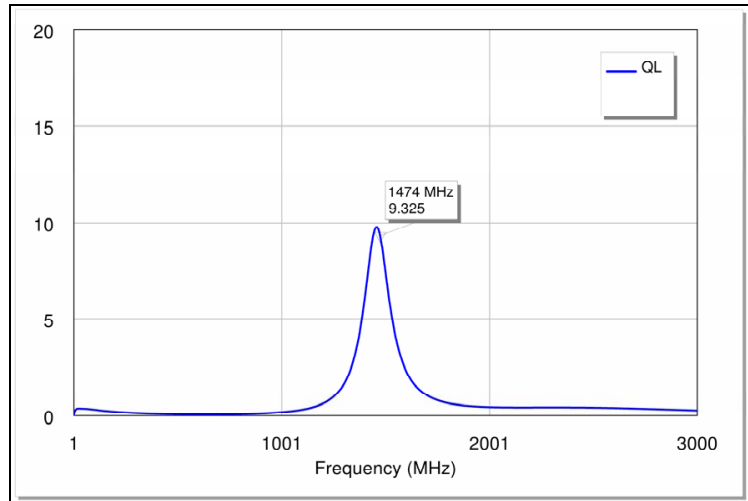


Figure 10. $Q_{L}$ of the common collector oscillator.

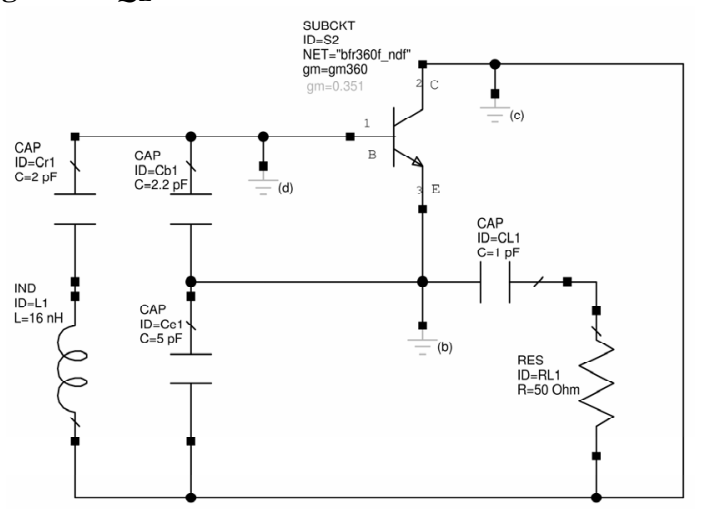

(a)

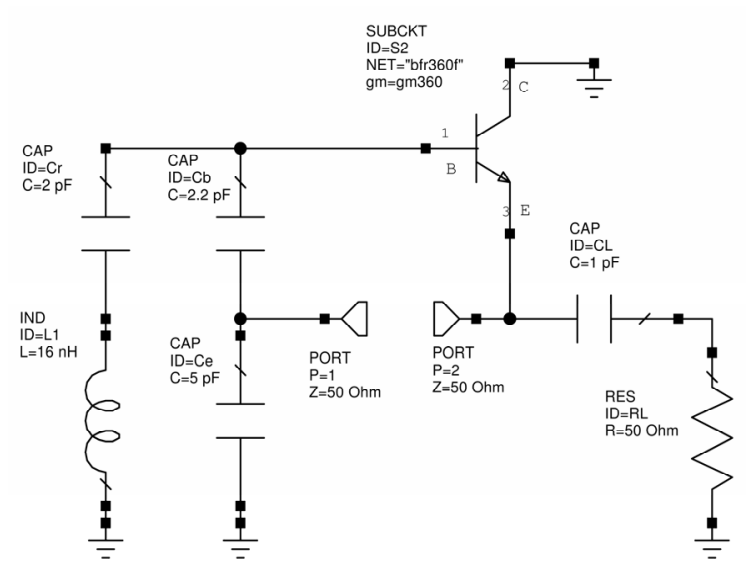

(c)

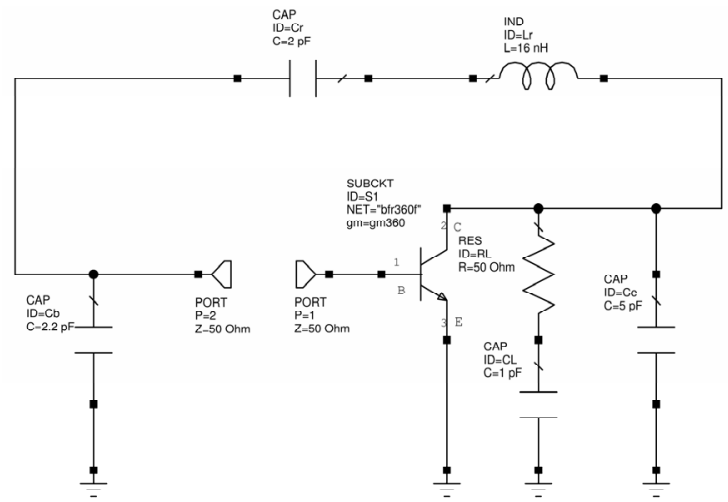

(b)

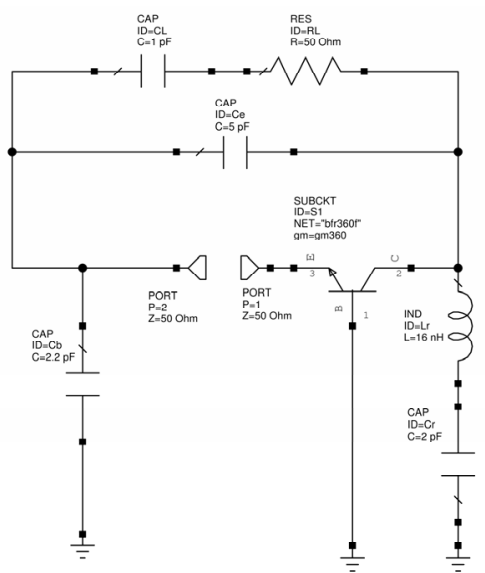

(d)

Figure 11. (a) Ground-less oscillator; (b) Common emitter oscillator; (c) Common collector oscillator; (d) Common base oscillator.

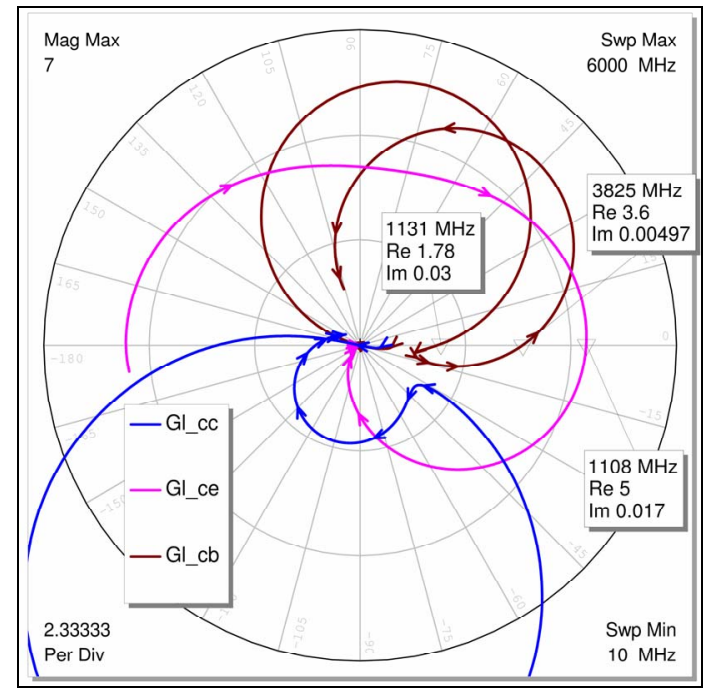

Figure 12. $G_{L}$ Nyquist plot for common emitter, common collector and common base.

three schematics. As it is expected, the solution for the three examples is the same, because they are the same circuit but drawn on three different ways.

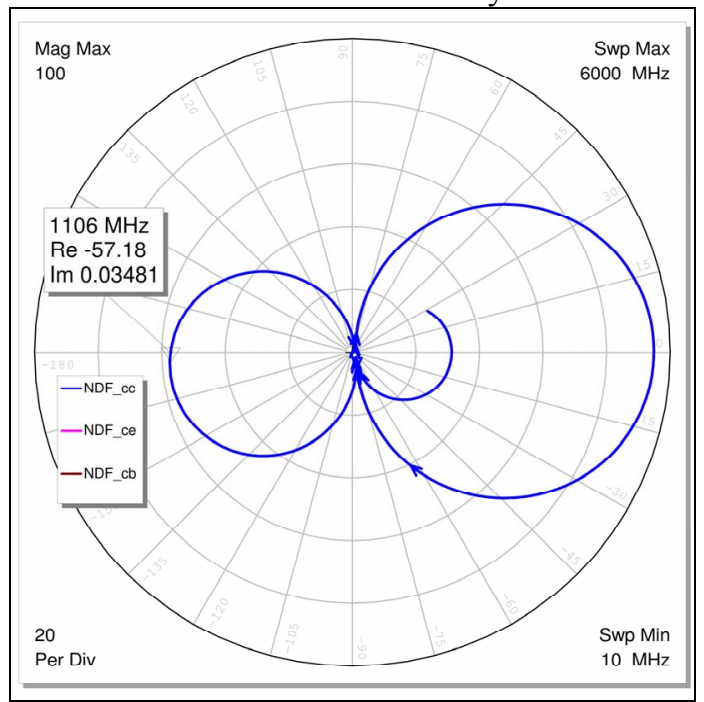

Figure 13. NDF Nyquist plot for the three circuit topologies.

\section{Conclusions}


The NDF method is a suitable tool for direct analysis of oscillators and it does not require any additional proviso or conditions before using it. Another advantage of this NDF method is that all oscillator topologies can be analyzed with a "loop-gain concept" and the main parameters that the reference plane methods cannot provide are now available for any oscillator topology.

The NDF solution is independent of the virtual ground position and it provides the oscillation frequency at first harmonic approximation without requiring transistor $g_{m}$ compression. This NDF independence is based on its relation with the Return Relations (and $R R_{T}$ ), as they provide the "true open-loop-gain". To sum up, the NDF/ $R R_{T}$ method is an optimum tool for the quasi-lineal oscillator analysis in a single step; it does not require any additional proviso or verification; and it is suitable for any oscillator topology.

\section{REFERENCES}

[1] D. J. Esdale and M. J. Howes, "A Reflection Coefficient Approach to the Design of One Port Negative Impedance Oscillators," IEEE Transactions on Microwave Theory and Techniques, Vol. 29, No. 8, 1981, pp. 770-776.

http://ieeexplore.ieee.org/xpl/articleDetails.jsp?arnumber $=1130445$

[2] S. Alechno, "Advancing the Analysis of Microwave Oscillators," Microwaves RF, Vol. 39, No. 6, 2000, pp. 5567.

[3] R. W. Rhea, "Discrete Oscillator Design: Linear, Nonlinear, Transient, and Noise Domains," Artech House Publishers, New York, 2010.

[4] R. W. Jackson, "Criteria for the on Set of Oscillation in Microwave Circuits," IEEE Transactions on Microwave Theory and Techniques, Vol. 40, No. 3, 1992, pp. 566569.

http://ieeexplore.ieee.org/xpl/articleDetails.jsp?arnumber $=121734$

[5] M. Randall and M. J. Hock, "General Oscillator Characterization Using Linear Open-Loop S-Parameters," IEEE Transactions on Microwave Theory and Techniques, Vol. 49, No. 6, 2001, pp. 1094-1100. doi:10.1109/22.925496

[6] V. González-Posadas, J. L. Jiménez Martín, A. ParraCerrada, D. Segovia-Vargas and L. E. García-Muñoz, "Oscillator Accurate Linear Analysis and Design. Classic Linear Methods Review and Comments," Progress in
Electromagnetics Research PIER, Vol. 118, 2011, pp. 89116.

http://www.jpier.org/PIER/pier.php?paper=11041403

[7] J. L. Jiménez-Martín, V. González-Posadas, A. ParraCerrada, L. E. García-Muñoz and D. Segovia-Vargas, "Comments and Remarks over Classic Loop-Gain Linear Method for Oscillators Design and Analysis. New Proposed Method Based on NDF/RRT," Radioengineering, Vol. 21, No. 1, 2012, pp. 478-491.

[8] J. L. Jiménez-Martín, V. González-Posadas, A. ParraCerrada, L. E. García-Muñoz and D. Segovia-Vargas, "Provisos for Classic Linear Oscillator Design Methods. New Linear Oscillator Design Based on the NDF/RRT," Progress in Electromagnetics Research PIER, Vol. 126, 2012, pp. 17-48. http://www.jpier.org/PIER/pier.php?paper=11112308

[9] A. Platzker and W. Struble, "Rigorous Determination of the Stability of Linear N-Node Circuits from Network Determinants and the Appropriate Role of the Stability Factor K of Their Reduced Two-Ports," 3rd International Workshop on Integrated Nonlinear Microwave and Millimeterwave Circuits, 1994, pp. 93-107.

$\mathrm{http}$ //ieeexplore.ieee.org/xpl/articleDetails.jsp?arnumber $=512515$

[10] R. W. Jackson, "Rollett Proviso in the Stability of Linear Microwave Circuits-a Tutorial," IEEE Transactions on Microwave Theory and Techniques, Vol. 54, No. 3, 2006, pp. 993-1000.

http://ieeexplore.ieee.org/xpl/articleDetails.jsp?arnumber $=1603843$

[11] M. Ohtomo, "Proviso on the Unconditional Stability Criteria for Linear Twoport," IEEE Transactions on Microwave Theory Tech, Vol. 43, No. 5, 1995, pp. 1197-1200. doi:10.1109/22.382086

[12] J. L. Jiménez Martín, V. González-Posadas, A. ParraCerrada, A. Blanco-del-Campo and D. Segovia-Vargas, "Transpose Return Relation Method for Designing Low Noise Oscillators," Progress in Electromagnetics Research PIER, Vol. 127, 2012, pp. 297-318. http://www.jpier.org/PIER/pier.php?paper=12022305

[13] S. Alechno, "The Virtual Ground in Oscillator DesignA Practical Example," Applied Microwave \& Wireless, Vol. 39, No. 7, 1999, pp. 44-53.

[14] N. M. Nguyen and R. G. Meyer, "Start-Up and Frequency Stability in High-Frequency Oscillators," IEEE Journal of Solid-State Circuits, Vol. 27, No. 5, 1992, pp. 810-820. doi:10.1109/4.133172 